\title{
La escritura femenina en lengua inglesa en el siglo XVII
}

\author{
MaRía del MAR Rivas CaRmona \\ Universidad de Sevilla
}

Fecha de recepción: 24 febrero 2007

Fecha de aceptación: 2 junio 2007

Resumen: Pese a las numerosas dificultades que encontraron, las mujeres del XVII consiguieron hacerse un hueco en un mundo literario tradicionalmente masculino. Entre otros problemas, las teorías que apoyaban una superioridad de los hombres en el ámbito intelectual llevaron a difundir el estereotipo de la escritora rebelde, insensata y desvergonzada. Siguiendo la estela de Aphra Behn, la primera escritora profesional, numerosas autoras comenzaron paulatinamente a contribuir con sus ingresos a la economía del propio hogar. Sus escritos se enmarcan en géneros muy diversos (Aughterson 1995), desde manuales del hogar y cartas personales a controversias políticas y obras dramáticas, pasando por poesía, prosa sentimental, ficción en prosa o escritos religiosos. El presente estudio pretende repasar y valorar la labor heroica y transgresora de las primeras mujeres, esas primeras voces "feministas" (Richards 1980), que decidieron que su papel no podía estar ligado exclusivamente a la aguja y al fogón.

Palabras clave: Literatura del XVII, mujeres escritoras, primeras feministas, géneros femeninos.

Abstract: In spite of the numerous obstacles they encountered, 17th century women managed to find a place for themselves in traditionally all-male literary circles. Among many other difficulties, the theories concerning men's intellectual superiority fostered the stereotype of a rebel, wild and impudent female writer. After Aphra Behn, the first professional female writer, many other women authors gradually began to contribute to the family finances with their literary income. Their writings belong to various discourse genres (Aughterson 1995), ranging from domestic handbooks and personal letters, to political controversies and drama, besides poetry, sentimental prose, prose fiction or religious writings. The aim of this study is to review briefly and evaluate the heroic and transgressive work of those first women, the first "feminist" voices (Richards 1980), who decided that their role was not exclusively tied to the needle and stew anymore.

Key words: 17th c. Literature, women writers, first feminists, female genres.

\section{INTRODUCCIÓN: LA BÚSQUEDA DE UNA VOZ PÚBLICA}

Los escritos en lengua inglesa que llevaron a cabo las mujeres durante el siglo XVII pertenecen a disciplinas discursivas tan variadas como el derecho, la teología, la medicina, la política, la historia y, por supuesto, la literatura. No resulta extraño, por tanto, que encontremos recopilaciones como la de K. Aughterson (1995) en la que se recogen manuscritos de tipo 
tan diverso como manuales del hogar, cartas de mujeres predicadoras $u$ osados prólogos de obras dramáticas ${ }^{1}$. Esta intensa labor, sin embargo, no estuvo carente de dificultades. De hecho, para la mayoría de las escritoras fue casi una hazaña poder escribir y, más aún, poder ver sus obras publicadas. Las mujeres que lograron realizar dichos objetivos tienen un incuestionable mérito.

Como señalaba Virginia Woolf en su conocida obra Una habitación propia (A Room of One's Own, 1929) ${ }^{2}$, con toda probabilidad, si Shakespeare hubiera tenido una hermana de enorme talento, jamás hubiera podido desarrollarlo; sin duda, el papel tradicional que tendría asignado estaría más cercano al fogón y a la aguja que al papel y la pluma:

\begin{abstract}
...it would have been impossible, completely and entirely, for any woman to have written the plays of Shakespeare in the age of Shakespeare. Let me imagine ... what would have happened had Shakespeare had a wonderfully gifted sister, called Judith, let us say. Shakespeare himself, went, very probably ... to the grammar school, where he may have learnt Latin -Ovid, Virgil and Horace- and the elements of grammar and logic... [He went] to seek his fortune in London... Meanwhile his extraordinarily gifted sister, let us suppose, remained at home. She was as adventurous, as imaginative, as agog to see the world as he was. But she was not sent to school, she had no chance of learning grammar and logic, let alone of reading Horace and Virgil. She picked up a book now and then, one of her brother's perhaps, and read a few pages. But then her parents came in and told her to mend the stockings or mind the stew and not moon about the books and papers... (Virginia Woolf, A Room of One's Own, ([1929] 1987:46)
\end{abstract}

\footnotetext{
${ }^{1}$ Kate Aughterson (ed.), Renaissance Women: Constructions of Femininity in England. New York: Routledge, 1995. Asimismo es muy interesante el volumen editado por CHEDGZOY, HANSEN y TRILL en el que se recogen trabajos presentados en el congreso Voicing Women celebrado en la Universidad de Liverpool en 1992 y que trata desde figuras tan afamadas como Aemilia Lanyer, Elizabeth Cary o Aphra Behn hasta escritoras menos reconocidas como Dionys Fitzherbert, Katherine Evans y Sarah Cheevers. En Kate Chedgzoy, Melanie Hansen y Suzanne Trill (eds.), Voicing Women: Gender and Sexuality in Early Modern Writing. Keele, Staffordshire: Keele UP, 1996.

${ }^{2}$ Virginia WoOLF, A Room of One's Own. London: Grafton Books, [1929] 1987.

3 "...habría sido absoluta y completamente imposible para cualquier mujer haber escrito las obras de Shakespeare en la época de Shakespeare. Me imagino ... lo que habría ocurrido si Shakespeare hubiese tenido una hermana de un enorme talento, llamada Judith, pongamos por caso. El propio Shakespeare, muy probablemente, fue ... a la escuela, donde seguramente aprendió latín -Ovidio, Virgilio y Horacio- y los elementos de la gramática y la lógica (...) [Fue a] buscar fortuna a Londres... Mientras tanto su extraordinariamente inteligente hermana, supongámoslo así, permanecía en casa. Era tan aventurera, tan imaginativa, estaba tan ansiosa por ver el mundo como él. Pero a ella no la enviaron a la escuela, no tuvo oportunidad de aprender gramática ni lógica, y mucho menos de leer a Horacio y Virgilio. De vez en cuando cogía un libro, quizá uno de los de su hermano, y leía unas
} 
Ya en el propio siglo XVII había voces femeninas que denunciaban tal discriminación y rechazaban ese papel tradicionalmente ligado a las faenas del hogar, al igual que la imagen poética de la mujer como una rosa, frágil y "callada". Son innumerables las ocasiones en las que las protagonistas de las narraciones femeninas aparecen bordando o cosiendo, de hecho.

Anne Finch, condesa de Winchilsea (1661-1720), rechazaba tajantemente dicha imagen femenina:

\author{
My hand delights to trace unusual things, \\ And deviates from the known and common way; \\ Nor will in fading silks compose \\ Faintly the inimitable rose... \\ (Anne Finch, 1661- 1720) ${ }^{4}$
}

En otro de sus trabajos, "Women and Fiction" ([1929] 1990), Virginia Woolf nos hacía caer en la cuenta de que, durante siglos, hemos conocido relatos interesantes de la vida de nuestros antepasados masculinos y, sin embargo, de nuestras abuelas y bisabuelas solamente sabíamos si eran guapas o si habían tenido mucha descendencia... una vida ligada al hogar y poco más. También se preguntaba en ese ensayo el motivo por el que no existió una continuidad en la labor literaria femenina anterior al siglo XVIII y hallaba la respuesta en el hecho de que las propias mujeres veían la escritura como una prerrogativa masculina. Como señalaban autoras como Crawford (1985: 214) ${ }^{6}$ y Spencer (1986: 26$)^{7}$, a la hora de referirse a las escritoras se recurría constantemente a términos "guerreros", puesto que la literatura era un "campo" masculino que accidentalmente "invadían" las mujeres.

cuantas páginas. Pero entonces llegaban sus padres y le decían que zurciera las medias o se ocupara del guiso y que no perdiese el tiempo con los libros y los papeles..." (Virginia Woolf, $A$ Room of One's Own, ([1929] 1987:46)) (Los subrayados, en este y en los ejemplos siguientes, son míos, así como las traducciones).

4 “ Mi mano se deleita en perseguir cosas inusitadas,

y se desvía de la forma conocida y común;

ni en sedas desteñidas componer puede

débilmente la rosa inimitable..." ".

(Anne Finch, 1661-1720)

Citada en Cora KAPLAN (1975:29) Salt, Bitter and Good: Three Centuries of English and American Women Poets. London, Paddington Press.

${ }^{5}$ Virginia Woolf, "Women and Fiction" ([1929]). En: Deborah Cameron (ed.), The Feminist

Critique of Language. A Reader. London: Routledge, 1990, pp. 33-40.

${ }^{6}$ Patricia CRAWFORD, "Women's published writings 1600-1700". En: Mary Prior (ed.) Women in English Society 1500-1800. London: Methuen, 1985, pp. 211-282.

${ }^{7}$ Jane SPENCER, The Rise of the Woman Novelist: from Aphra Behn to Jane Austen. Oxford: Blackwell, 1986. 
Las propias autoras consideraban su faceta literaria como su "parte masculina". Así, le ocurría a Aphra Behn que hablaba de "my Masculine Part the Poet in $\mathrm{me}^{\prime \prime}$.

De forma absolutamente irónica la antes mencionada Anne Finch, condesa de Winchilsea, reclamaba a tres poetas contemporáneos que ellos habían nacido para escribir y mandar, en tanto que las mujeres, "ceros a la izquierda", sólo habían nacido para engendrarlos y deleitarlos:
Happy you three! Happy the Race of Men!
Born to inform or to correct the Pen
To proffitts pleasures freedom and command
Whilst we beside you but as Cyphers stand
T'increase your Numbers and to swell th'account
Of your delights which from our charms amount
And sadly are by this distinction taught
That since the Fall (by our seducement wrought)
Ours is the greater losse as ours the greater fault. ${ }^{9}$

Todas estas limitaciones llevaron a que, cuando se decidieron a escribir, convirtieran su hogar y su vida personal en el objeto literario en la mayoría de las ocasiones: "...female creativity has had to express itself within the confines of domesticity"10. Por supuesto, si estas mujeres intentaban salir de estos confines de lo doméstico y pasaban a reivindicar una voz pública recibían irremisiblemente un torrente de críticas mordaces. Así lo expresaba, de forma irónica una vez más, Margaret Cavendish, duquesa de Newcastle: "Work Lady work, let writing Books alone / For surely wiser Women nere wrote one". ${ }^{11}$ Similares son las palabras de Lady Winchilsea:

\footnotetext{
${ }^{8}$ "Mi parte masculina el poeta que llevo dentro". Citado por Sandra M. Gilbert, Sandra M. y Susan Gubar, The Madwoman in the Attic.The Woman Writer and the Nineteenth-Century Literary Imagination. New Haven: Yale University Press, 1979, p. 66, nota 40.

${ }^{9}$ Citado en Gilbert y Gubar (1979:8).

¡Vosotros tres, sed felices! ¡Raza de los Hombres, sé feliz!

Nacida para informar o la Pluma corregir,

para los beneficios, los placeres, la libertad y el mando,

mientras nosotras como ceros a la izquierda a vuestro lado nos sentamos

para incrementar vuestro Número y para aumentar el recaudo

de vuestros deleites que se suman de nuestros encantos

y que, tristemente, aprenden por esta distinción

que desde la Caída (provocada por nuestra seducción) nuestra es la mayor culpa y nuestra la mayor perdición.

10 "...la creatividad femenina ha tenido que expresarse dentro de los confines de lo doméstico".

11 "Trabaja, Mujer, trabaja, deja los Libros en paz / porque seguramente mujeres más sabias no escribieron ninguno jamás". (Citada por Crawford (1985:276). El énfasis es de la autora).
} 
Alas! A woman that attempts the pen,

Such an intruder on the right of men,

Such a presumptuous creature is esteemed,

The fault can by no virtue be redeemed. $(1661)^{12}$

They tell us, we mistake our sex and way;

Good breeding, fassion, dancing, dressing,

Are the accomplishments we should desire;

To write, or read, or think, or to inquire

Would cloud our beauty, and exhaust our time,

And interrupt the conquests of our prime;

Whilst the dull manage of a servile house

Is held by some, our utmost wit and use. ${ }^{13}$

Casi ninguna escritora contó con el apoyo familiar (salvo alguna aristócrata como Margaret Cavendish que sí tenía el apoyo de su esposo). De hecho, el dedicarse a escribir conllevaba la pérdida de la reputación propia y la de toda la familia. Prueba de ello son las duras y ofensivas palabras que dirigía el hermano de Elizabeth Avery a la autora tras la publicación de un trabajo de ésta en 1647: "your printing of a Book, beyond the custom of your Sex, doth rankly smell" ${ }^{14}$. En el mejor de los casos se las ignoraba o no se las tomaba en serio, aunque lo normal era que se las criticara y se las ridiculizara.

El lugar y el papel de las mujeres estaba nítidamente perfilado en esa sociedad, era una esfera aparte; en palabras de Crawford (1985: 214):

\footnotetext{
${ }^{12}$ ¡Ah! Una mujer que pretende escribir, en los derechos de los hombres tal intrusa, es considerada tal criatura presuntuosa,

que la falta bajo ningún motivo se puede redimir. (1661)

Anne FINCH (1928) 'The Introduction', Miscellany Poems on Several Occasions. Ed. J.M. Murry. London, Jonathan Cape. (También citada por Virginia Woolf ([1929] 1987:57)).

${ }^{13}$ Nos dicen que nos equivocamos de sexo y de manera; buena crianza, buen gusto, bailar, vestir bien,

son los logros que deberíamos desear;

escribir, o leer, o pensar, o preguntar

ensombrecerían nuestra belleza, y agotarían nuestro tiempo,

e interumpirían las conquistas de nuestro mejor momento;

mientras el aburrido gobierno de una casa servil

es para algunos, nuestro mayor talento y utilidad.

Citado en S.M. GILBERT \& S. GUBAR (1979:8); también en Woolf ([1929] 1987:57) y en el prólogo de Michèle Barrett (ed.) ([1979] 1981) Virginia Woolf: Las mujeres y la literatura. Barcelona, Lumen. (Prólogo y selección de M. Barrett).

14 "el que publiques un Libro, más allá de la costumbre de tu Sexo, huele demasiado mal" (citado en Crawford (1985:217)).
} 
"Their place was the household. Ideally, a good woman was modest and silent...". ${ }^{15}$ Hasta no hace muchos años, de hecho, se ha seguido asociando la esfera privada con la esfera femenina, en tanto la esfera pública ha sido el ámbito masculino. Como señala Clare $(1996)^{16}$, el gran problema para las mujeres del XVII comenzaba cuando pretendían trascender la esfera privada en la que se las había circunscrito. La escritora Anne Finch lo expresaba con estos versos:

Did I my lines extend for publick view,

How many censures, wou'd their faults persue,

Some wou'd, because such words they do affect,

Cry they're insipid, empty, uncorrect.

And many, have attain'd, dull and untaught

The name of Witt, only by finding fault.

True judges, might condemn their want of witt,

And all might say, they're by a Woman writt. ${ }^{17}$

También la autora Dorothy Leigh se quejaba de las grandes dificultades que encontraban para poder publicar sus obras; en 1616, en un libro de consejos a las madres, afirmaba que escribir era casi insólito para una mujer ("something unusual among us") ${ }^{18}$. Como afirma Crawford (1985: 226), las mujeres del XVII sabían que no se las consideraba igual que a los hombres de su mismo rango, pero, por eso mismo, querían denunciarlo:

In seventeenth-century England, women knew that their ideas were not so highly valued as those of men of the same social rank. Nevertheless, because some knew that their experiences as women were dissimilar from those of men, they wrote to bear witness to their own different reality. ${ }^{19}$

\footnotetext{
15 "Su lugar era el hogar. Idealmente, una buena mujer era modesta y callada...".

16 Janet CLARE, "Transgressing Boundaries: Women's Writing in the Renaissance and Reformation." Renaissance Forum, 1,1, 1996.

${ }^{17}$ A extender mis versos al ámbito público me atreví,

cuántas censuras, sus faltas habrán de recibir,

algunos, como esas palabras les afectan,

Gritarán que son insulsas, vacías e incorrectas.

Y muchos, han conseguido, aburrido y no enseñado

el nombre de Talento, solamente errores hallando.

Como verdaderos jueces su falta de ingenio condenarían,

y, que están escritos por una mujer, todos repetirían.

(Anne Finch. Citada por Dale Spender (1985:195).

18 "algo inusitado entre nosotras". Dorothy Leigh (1616) The Mothers Blessing. Citada por Patricia Crawford (1985:212)

${ }^{19}$ En la Inglaterra del siglo XVII, las mujeres sabían que sus ideas no eran tan altamente valoradas como las de los hombres de su mismo rango social. No obstante, como algunas eran conscientes
} 
Como consecuencia de todo esto, la temática más habitual en sus escritos era, por un lado, la denuncia de la tremenda desigualdad social de la época y la expresión de su deseo de justicia, y, por otro, la exposición de experiencias que pudieran servir a otras mujeres a través de consejos o del relato de sus propias vidas (su matrimonio, la maternidad...) ${ }^{20}$. De ahí que los primeros géneros que emplearon las mujeres como vía de expresión fueran las cartas, los tratados morales, los artículos de interés doméstico o las novelas para otras mujeres. ${ }^{21}$

\section{PRIMERAS VOCES "FEMINISTAS"}

Para Janet Richards (1980:13) ${ }^{22}$ estas primeras escritoras se pueden considerar verdaderas "feministas", puesto que empleaban sus obras como vehículo de denuncia de la marginación de la mujer y de su confinamiento en el ámbito de lo privado. Es más, el simple hecho de dedicarse a la escritura ya las convertía en combatientes contra el status quo que las pretendía restringir a una esfera silenciada. Aunque ninguna tuviera conciencia de esa labor "feminista", al cuestionarse su papel en la sociedad e identificarse como grupo oprimido estaban poniendo las bases de la teoría feminista moderna (Hilda L. Smith 1982) ${ }^{23}$.

No son pocos los ejemplos de reivindicaciones de este tipo. Así, Aemilia Lanyer culpaba a Adán y no a Eva de la Caída y negaba de plano que el hombre fuese superior a la mujer:

Then let vs haue our Libertie againe,

And challendge to your selues no Sou'raigntie;

You came not in the world without our paine,

Make that a barre against your crueltie.

(Salve deus rex ludaeorum, 1611) ${ }^{24}$

de que sus experiencias como mujeres eran distintas de las de los hombres, escribieron para dejar constancia de su diferente realidad. (Crawford 1985:226)

${ }^{20}$ Hubo mujeres que se sintieron impulsadas a escribir porque pensaban que seguían designios divinos, como ocurrió con muchas cuáqueras (CRAWFORD 1985:224). Hilary HINDS trata este problema con minuciosidad en God's Englishwomen: Seventeenth-Century Radical Sectarian Writing and Feminist Criticism. Manchester: Manchester UP, 1996.

${ }^{21}$ El público femenino era el mayor lector de novelas durante los siglos XVIII y XIX.

22 Janet RICHARDS, The Sceptical Feminist: A Philosophical Enquiry. London: Virago, 1980.

${ }^{23}$ Hilda L. SMITH, Reasons's Disciples: Seventeenth-Century English Feminists. Urbana, University of Illinois Press, 1982.

${ }^{24}$ Devolvednos entonces nuestra Libertad,

y no desafiéis ninguna Soberanía;

no vinisteis al mundo sin nuestro dolor,

haced de eso un freno contra vuestra Tiranía.

(Salve deus rex ludaeorum, 1611)

Cfr. Crawford (1985:228). 
Elizabeth Johnson, impresora, reivindicaba en 1690 los derechos de la mujer y tachaba de "violación de la libertad de la mujer" al monopolio de la expresión pública por parte de los hombres. ${ }^{25}$

Margaret Cavendish, duquesa de Newcastle, reivindicaba el acceso de la mujer a la educación y al empleo.

La autora anónima de Triumphs of Female Wit (1683) denunciaba que el hogar se había convertido en la prisión de la mujer y describía el destino de su vida como coser y cocinar ("needles and puddings") ${ }^{26}$.

Muchas otras contemporáneas de ellas estaban convencidas de que no era un hecho inconsciente por parte de los hombres el querer mantenerlas débiles e ignorantes. Ellas sabían que el primer paso para lograr mayores derechos sería conseguir el derecho a la educación.

\section{LAS FORMAS DE EXPRESIÓN PERSONAL}

Aunque los primeros libros que se imprimieron en Inglaterra son del siglo XV, hasta el siglo XVII no aparecen publicaciones escritas por mujeres, por otra parte, muy mal acogidas por la sociedad (Crawford 1985:211). Las obras escritas por mujeres en el período de las Guerras Civiles y el Interregno fueron en su mayoría ignoradas por sus contemporáneos o, incluso, se vieron abocadas al olvido o atribuidas a otros autores masculinos.

Los temas y géneros que abordaban las mujeres en estas primeras publicaciones eran de diversa índole: poesía ${ }^{27}$, teatro, ficción en prosa, prosa sentimental, escritos religiosos, controversias políticas... De este último ámbito eran muchos de los escritos que las mujeres hacían durante las Guerras Civiles para defender a sus esposos o pedir su liberación y, casi siempre, encontraban negativas despectivas como demuestra el siguiente escrito de abril de 1649:

...That the matter you petition about, is of an higher concernement than you understand. That the House gave an answer to your husbands; and therefore that you are desired to goe home, and looke after your owne businesse, and meddle with your huswifery.

(Citado por Crawford 1985:213)

\footnotetext{
25 "Violations on the Liberties of Free-born English Women": "Violaciones de las Libertades de las Mujeres Inglesas Nacidas Libres" (Ibidem, p..224).

${ }^{26}$ Ibidem, p. 228

${ }^{27}$ Véase Bronwen Price, "Women's poetry 1550-1700: 'Not unfit to be read'. En: Anita PACHECO (ed.), A companion to Early Modern Women's Writing. Oxford: Blackwell, 2002, pp. 282-302.

${ }_{28}^{2 . . . Q u e ~ e l ~ a s u n t o ~ d e l ~ q u e ~ h a c e ́ i s ~ p e t i c i o ́ n ~ e s ~ d e ~ m a y o r ~ t r a s c e n d e n c i a ~ d e ~ l a ~ q u e ~ v o s o t r a s ~ e n t e n d e ́ i s . ~}$ Que el Consejo ha dado una respuesta a vuestros esposos; y que, por tanto, se os pide que os vayáis a casa, y os preocupéis de vuestras cosas, y os dediquéis a vuestros asuntos domésticos.
} 
Las mujeres, acostumbradas a las formas de expresión personal de carácter privado, optaron por desarrollarlas como medio de expresión ante las dificultades para escribir otro tipo de publicaciones. Así, las cartas, las autobiografías o los diarios se convirtieron en el reflejo de "una vida experimentada como un arte o de una experiencia artística como forma de vida" ("life experienced as an art or an art experience as a kind of life") (Susan Gubar, 1982: 82).

Incluso cuando se dedicaban a escribir obras dramáticas lo hacían, en la mayoría de las ocasiones, sin ninguna pretensión pública. Los motivos que las llevaban a escribir teatro eran puramente privados, bien como una práctica educativa o bien para el entretenimiento de la familia o de un grupo reducido de amigos (Cesarano y Wynne-Davies 1996; Tomlinson 2002). ${ }^{29}$ La primera autora inglesa que publicó un drama original fue Elizabeth Cary con The Tragedy of Mariam ${ }^{30}$ de 1613. También es digna de mención la labor dramática de Lady Mary Wroth. Su tragicomedia Love's Victory (c. 1620), aunque sólo fue representada o leída ante un pequeño auditorio, cuenta con acotaciones que hacen pensar que tuviera intención de presentarla públicamente.

Así pues, resultaba anecdótico que las mujeres escribieran libros con verdaderos propósitos de reconocimiento público. Como señalaba Virginia Woolf $(1967: I I I, 60)^{31}$, el propio hecho de escribir libros resultaba ya ridículo para las mujeres en el siglo XVII. No obstante, las formas de expresión más íntima como las cartas no eran consideradas indecorosas. De ese modo el silencio se fue rompiendo poco a poco:

Had she been born in 1827, Dorothy Osborne would have written novels; had she been born in 1527, she would never have written at all. But she was born in 1627, and at that date though writing books was ridiculous for a woman there was nothing unseemingly in writing a letter. And so by degrees the silence is broken...

La mayoría de las autoras del siglo XVII de quienes han perdurado obras en prosa son conocidas por sus cartas o sus autobiografías. Las más

\footnotetext{
${ }^{29}$ S. P. Cerasano y Marion WYNNE-DAVIES (eds), Renaissance Drama by Women: Texts and Documents. Nueva York: Routledge, 1996. Sophie TOMLINSON, "Drama". En: Anita PACHECO (ed.), A companion to Early Modern Women's Writing. Oxford: Blackwell, 2002, pp. 317-335.

30 Aunque Cary mantiene las tres unidades dramáticas (acción, tiempo y lugar), siguiendo la obra original de Séneca en la que se basaba, profundiza en el papel de Miriam frente al de Herodes.

${ }^{31}$ Virginia WooLF, "Dorothy Osborne's Letters", Collected Essays. London, Chatto \& Windus, 1967.

${ }^{32}$ Si hubiese nacido en 1827, Dorothy Osborne habría escrito novelas; si hubiese nacido en 1527, no habría escrito nunca nada en absoluto. Pero nació en 1627, y en esa fecha, aunque escribir libros era ridículo en una mujer, no había nada indecoroso en escribir una carta. Y de esta forma, paso a paso, se fue rompiendo el silencio...
} 
destacadas fueron Lady Brilliana Harley (1600-1643), Margaret Cavendish, duquesa de Newcastle (1624-1674), Katherine Philips (1631-1664), Dorothy Osborne (1627-1695) y, por supuesto, Aphra Behn (1640-1689) ${ }^{33}$, entre otras.

No cabe duda de que las cartas como formas de expresión personal constituyeron una base importante en la transición hacia la novela, género femenino por antonomasia en sus orígenes y que, esencialmente, suponía una expresión de la experiencia del individuo. Asimismo, la autobiografía está íntimamente relacionada con el desarrollo de la novela, puesto que también permitía a las autoras narrar sus experiencias vitales ${ }^{34}$. La primera autobiografía que se conserva es de 1373 y es la vida de Margery Kempe, que tuvo que dictarla pues no sabía escribir (Donovan 1990:48). En el siglo XVII destacaron Alice Thornton (1627-1707), la duquesa de Newcastle, la condesa de Warwick (1624-1678), Lady Fanshawe (1625-1680), Lady Giffard (1639-1722) o Mary de la Rivière Manley (1663-1724) (que posteriormente alcanzó gran fama como novelista).

No existe una equivalencia clara entre las memorias que se escribían en el XVII y las autobiografías o diarios actuales, puesto que entonces las memorias suponían un verdadero compendio de estilos y temas, que incluían manuscritos de tipo variopinto desde diarios, meditaciones, instrucciones para los hijos, recuerdos autobiográficos, consejos, oraciones, comentarios sobre la Biblia, etc., como señala Mendelson (1985:182).

Tampoco podemos hablar de un prototipo de mujer dedicada a la escritura de memorias, pues las autoras pertenecen a todos los estados civiles y edades. Las de Lady Elizabeth Delaval comienzan cuando ella cuenta con catorce años y el diario de Sarah Savage llega hasta sus ochenta años, por ejemplo. Sin embargo, coinciden en pertenecer a una élite reducida que sabía escribir; de hecho, de veintitrés diarios conocidos diecisiete pertenecen a la clase alta o aristócrata, seis a la clase media y ninguno a la clase baja. Por aquellos entonces, aunque muchas mujeres sí sabían leer, la mayoría no sabía escribir, ni tan siquiera firmar. Como afirma Cressy (1980:147), el porcentaje de mujeres analfabetas en la época de los Estuardo estaba entre un 84 y un $98 \%$. En Londres las cifras eran de un $78 \%$ de analfabetas en 1670 , un $64 \%$ en 1680 y un $52 \%$ en $1690{ }^{35}$.

Pese a que las mujeres escribían con un público in mente, muchas de ellas no tenían claras ambiciones públicas; por el contrario, en el diario expresaban sus más íntimas quejas sobre su vida o sus maridos, de aquí que un buen número de ellos tuvieran unas claves taquigráficas inventadas

\footnotetext{
${ }^{33}$ Aphra Behn fue la primera escritora profesional.

${ }^{34}$ Sobre la autobiografía véase Sheila OTTWAY, "Autobiography". En: Anita PACHECO (ed.), A companion to Early Modern Women's Writing. Oxford: Blackwell, 2002, pp. 231-247.

${ }^{35}$ D. CRESSY (1980:147) Literacy and the Social Order. Cambridge, C.U.P.
} 
por ellas mismas para evitar que sus esposos los leyeran ${ }^{36}$. No es de extrañar que muchos de los manuscritos hayan desaparecido, por tanto, puesto que la familia no los entendía y los tiraba o porque se avergonzaban de ellos, o, incluso, porque ellas mismas los destruían cuando enfermaban por miedo a que los descubrieran: "In writing to themselves... women could create a private place in which to speak the unthought, unsaid and undervalued." (Simons 1990: 27$)^{37}$.

A través de los diarios las mujeres señalaban tres etapas fundamentales en su vida y las tres giraban en torno a su relación con los hombres: soltería, matrimonio y viudedad. Coinciden en describir la primera etapa como la mejor y el matrimonio como el punto de inflexión crucial, que en muchas ocasiones podía constituir un verdadero trauma (Mendelson 1985:192). Otros temas estaban relacionados con la maternidad: el embarazo, el parto, los hijos... También recogían estos diarios autoconfesiones de claro fervor religioso al más puro estilo puritano. Los editores, de hecho, preferían publicar los diarios de tipo devocional.

Un tema en común en todas estas memorias es la detallada descripción de la vida doméstica. Gracias a los diarios sabemos, por ejemplo, que no existían grandes diferencias entre las actividades cotidianas de las mujeres de distinta clase social: la lectura (especialmente libros de tema religioso o sentimental), la costura, la educación de los hijos, las visitas a la familia o amistades, la beneficencia...

\footnotetext{
${ }^{36}$ Por ejemplo, Elizabeth Walker hizo prometer a su esposo que jamás se volvería a acercar a su diario. Samuel Pepys, esposo de Elizabeth Pepys, escribió lo siguiente acerca del diario de su esposa:

...so picquant, and wrote in English and most of it true, of the retiredness of her life and how unpleasant it was, that being writ in English and so in danger of being met with and read by others, 1 was vexed at it and desired her and then commanded her to teare it... (citado en Mendelson 1985:184)

...tan picante, y escrito en inglés y en su mayor parte verdadero, acerca del carácter retirado de su vida y de lo insatisfactoria que era, que estando escrito en inglés y tan en peligro de ser encontrado y leído por otros, me sentí enfadado por él y le pedí y luego le ordené que lo rompiese...

Otra escritora, Katherine Austen, advertía a cualquiera que pudiere encontrarse con su diario:

Who so ever shal look in these papers ... will easily discern it concerned none but my self \& was a private exercise directed to my self... The singularity of these conceptions doth not advantage any. (ibidem)

El que alguna vez leyese estos papeles... se dará perfecta cuenta de que no han tratado de nadie más que de mí y que han sido un ejercicio privado dirigido a mí misma... La singularidad de estas ideas no beneficia a nadie.

37 "Al escribir para ellas mismas... las mujeres podían crear un espacio privado en el que expresar lo no pensado, lo callado y lo subestimado". (F.Nussbaum citada por Judy SIMONS (1990: 27) Diaries and Journals of Literary Women from Fanny Burney to Virginia Woolf. London, Macmillan.
} 
Todas estas inquietudes de carácter privado encontrarán cauce de continuación en la novela.

Como señala Davies $(1981: 58-80)^{38}$, los diarios escritos por los hombres tenían un carácter bien diferenciado, pues no respondían a necesidades de índole privada, sino pública; tendían a narrar sucesos políticos, sus actividades laborales, etc.

\section{EL TRIUNFO DEL "ESTILO LLANO"}

A partir de la mitad del siglo XVII, en concreto alrededor de $1660^{39}$, se fecha el triunfo del "estilo llano", característico de la novela y que hereda de los subgéneros de transición como cartas, memorias y diarios las principales características de estos medios de expresión personal: el tono autobiográfico, esencialmente en primera persona narrativa ${ }^{40}$, y la descripción de la vida cotidiana y las labores domésticas. Autoras como Dorothy Osborne o Margaret Cavendish expresaron públicamente su satisfacción por poder, al fin, emplear un estilo adecuado para las mujeres; ésta última lo expresaba con los siguientes versos:

Give me the style that Nature frames, not art,

For art doth seem to take the pedant's part;

And what seems noble, which is easy, free,

Not to be bound with o'er-nice pedantry.

(Poems and Fancies, 1653)

Margaret Cavendish, duquesa de Newcastle (1623-1673), es una de las escritoras que logró gran fama en su época. Publicó su obra por placer, porque como aristócrata tenía una situación acomodada; además, como ella misma explicaba: "Sólo deseo la fama" (citado por V. Woolf ([1911] $1981: 93))^{42}$.

\footnotetext{
${ }^{38}$ Kathleen DAVIES, "Continuity and Change in Literary Advice on Marriage", págs.58-80, en R. Outhwaite (ed.) Marriage and Society. New York, Praeger, 1981.

${ }^{39}$ En Robert Adolph, The Rise of Modern Prose Style. Cambridge (Mass.), MIT Press, 1968.

${ }^{40} \mathrm{La}$ autobiografía no era únicamente característica del estilo femenino, también otros escritores como Defoe la empleaban con asiduidad (WATT 1957:15).

${ }^{41}$ Dadme el estilo que foria la Naturaleza, no el arte,

Pues el arte parece tomar la parte del pedante;

Y lo que parece noble, que fácil, libre, sería,

No se ha de relacionar con la remilgada pedantería.

(Poems and Fancies, 1653)

Citado por DonOvan (1990:50-51).

${ }^{42}$ Frente a estas autoras que buscaban la fama se hallaban escritoras como Katherine Philips, a la que la publicación no autorizada de sus poemas en 1664 le costó una enfermedad (DONOVAN 1990:47).
} 
También obtuvo gran renombre en la segunda mitad del XVII Aphra Behn (1640-1689), quien, al igual que ocurriera con Mary Delarivière Manley (1671-1724), comenzó escribiendo teatro, pero se decantó por la novela que, no sólo era un nuevo estilo que triunfaba, sino que también proporcionaba mayores ventajas económicas ${ }^{43}$. De hecho, Aphra Behn, la primera escritora profesional de Inglaterra, tenía que escribir para poder sustentarse tras haber enviudado. Virginia Woolf opinaba que con ella había comenzado la libertad de pensamiento para la mujer: "here begins the freedom of the mind" ([1929] 1987:61). Sin embargo, en su época era considerada "a shady lady" ("una dama turbia"), absolutamente indecente por su dedicación a la literatura, y la excluyeron de las bibliotecas y del canon literario.

Aphra Behn no fue la única en ser tachada de "atípica". A Margaret Cavendish también la denominaban "la loca Madge" ("Mad Madge") por su valentía en su actividad literaria. A Anne Finch, Lady Winchilsea, la tildaban de loca, rebelde y soberbia. Ser mujer y escritora equivalía por el momento a ser poco menos que un monstruo falto de razón y de femineidad.

Para las últimas décadas de siglo con la desaparición del patronazgo y de las formas privadas de circulación, se empezaba a abrir el terreno a las mujeres. Entre 1660 y 1800 ya más de un tercio de las publicaciones en prosa eran obra de escritoras ${ }^{44}$, en su mayor parte novelas sentimentales en forma epistolar. En 1692 aparecía incluso una revista exclusivamente femenina, el Ladies Mercury.

\section{CONCLUSIÓN}

Gracias a su esfuerzo las mujeres lograron hacerse un destacable hueco en el terreno literario durante el siglo XVII, aunque durante mucho tiempo siguieron encontrando numerosas dificultades. Diversas teorías ampararon durante este siglo y el posterior la creencia en un poder de raciocinio superior en los hombres que condujo a la creación del estereotipo de la mujer escritora como una loca desvergonzada, procaz e indecente.

Sin embargo, siguiendo la estela de Aphra Behn, la primera escritora profesional, se adentraron en un mundo hasta entonces exclusivamente masculino y para comienzos del XVIII eran cientos las mujeres que contribuían a la economía doméstica con sus ingresos literarios.

Las autoras que protagonizan la presente antología son una pequeña, aunque significativa muestra de la prodigiosa labor llevada a cabo por unas mujeres que sembraban en terreno poco abonado para sus fines, pero que

\footnotetext{
${ }^{43}$ Las motivaciones económicas también se encontraban en autoras como Elizabeth Channel, analfabeta y pobre, a la que Arise Evans ayudaba a escribir (CRAWFORD 1985:214).

${ }^{44} 265$ de las 760 entradas de The New Cambridge Bibliography of English Literature. Consúltese George WATSON (ed.), (1971), vol. 2, Cambridge, C.U.P.
} 
consiguieron que fructificaran las simientes que plantaron en forma de dramas, relatos, cartas o poemas.

REFERENCIAS BIBLIOGRÁFICAS

ABEL, Elizabeth (ed.), Writing and Sexual Difference. Chicago: Univ. of Chicago Press, 1982.

AdoLPH, Robert, The Rise of Modern Prose Style. Cambridge (Mass.): MIT Press, 1968.

Aughterson, Kate (ed.), Renaissance Women: Constructions of Femininity in England. New York: Routledge, 1995.

BARRETT, Michèle (ed.), Virginia Woolf: Las mujeres y la literatura. Barcelona: Lumen, [1979] 1981.

Cameron, Deborah (ed.), The Feminist Critique of Language. A Reader. London: Routledge, 1990.

Cerasano, S. P. y Marion Wynne-Davies, eds. Renaissance Drama by Women: Texts and Documents. New York: Routledge, 1996.

ChedgzoY, Kate, Melanie Hansen y Suzanne Trill (eds.), Voicing Women: Gender and Sexuality in Early Modern Writing. Keele, Staffordshire: Keele UP, 1996.

CLARE, Janet, "Transgressing Boundaries: Women's Writing in the Renaissance and Reformation." En: Renaissance Forum, 1, 1, 1996.

CraWFORD, Patricia, "Women's published writings 1600-1700". En: Mary Prior (ed.) Women in English Society 1500-1800. London: Methuen, 1985, pp. 211-282.

CRESSY, David, Literacy and the Social Order. Cambridge: Cambridge University Press, 1980.

DAVIES, Kathleen, "Continuity and Change in Literary Advice on Marriage". En: R. Outhwaite (ed.) Marriage and Society. New York: Praeger, 1981, pp. 58-80.

Donovan, J. "The silence is broken". En: Deborah Cameron (ed.) The Feminist Critique of Language. A Reader. London: Routledge, 1990, pp. 41-56. (Orig. en MacConnell-Ginet et alii (1980))

FINCH, Anne, 'The Introduction', Miscellany Poems on Several Occasions. Ed. J.M. Murry. Londres, Jonathan Cape, [16??] 1928.

GILBERT, Sandra M. y Susan Gubar, The Madwoman in the Attic. The Woman Writer and the Nineteenth-Century Literary Imagination. New Haven: Yale University Press, 1979.

GUBAR, Susan, “The Blank Page' and the Issues of Female Creativity". En: Elizabeth Abel (ed.) Writing and Sexual Difference. Chicago: Univ. of Chicago Press, 1982, pp. 73-93.

HINDS, Hilary, God's Englishwomen: Seventeenth-Century Radical Sectarian Writing and Feminist Criticism. Manchester: Manchester UP, 1996. 
KAPLAN, Cora, Salt, Bitter and Good: Three Centuries of English and American Women Poets. London: Paddington Press, 1975.

McConNELL-GINET, Sally, Women and Language in Literature and Society. New York: Praeger, 1980.

MendeLSON, S. H., "Stuart women's diaries and occasional memoirs". En: Mary Prior (ed.) Women in English Society 1500-1800. London: Methuen, 1985, pp. 181-210.

OTTWAY, Sheila, "Autobiography". En: Anita Pacheco (ed.), A companion to Early Modern Women's Writing. Oxford: Blackwell, 2002, pp. 231-247.

PACHECO, Anita (ed.), A companion to Early Modern Women's Writing. Oxford: Blackwell, 2002.

PrICE, Bronwen, 'Women's poetry 1550-1700: 'Not unfit to be read'. En: Anita Pacheco (ed.), A companion to Early Modern Women's Writing. Oxford: Blackwell, 2002, pp. 282-302.

PRIOR, Mary (ed.) Women in English Society 1500-1800. London: Methuen, 1985.

RICHARDS, Janet, The Sceptical Feminist: A Philosophical Enquiry. London: Virago, 1980.

SIMONS, Judy, Diaries and Journals of Literary Women from Fanny Burney to Virginia Woolf. London: Macmillan, 1990.

SMITH, Hilda L., Reasons's Disciples: Seventeenth-Century English Feminists. Urbana: University of Illinois Press, 1982.

SPENCER, Jane, The Rise of the Woman Novelist: from Aphra Behn to Jane Austen. Oxford: Blackwell, 1986.

SPENDER, Dale, Man-Made Language, London: Routledge \& Kegan Paul, [1980] rev.1985.

TOMLINSON, Sophie, "Drama". En: Anita Pacheco (ed.), A companion to Early Modern Women's Writing. Oxford: Blackwell, 2002, pp. 317-335.

WATSON, George, (ed.) The New Cambridge Bibliography of English Literature. Vol. 2, Cambridge, C.U.P., 1971.

WATT, Ian, The Rise of the Novel: Studies in Defoe, Richardson and Fielding. Berkeley: University of California Press, 1957.

Woolf, Virginia, "La Duquesa de Newcastle". En: Michèle Barrett (ed.) (1981) Las mujeres y la literatura. Barcelona: Lumen, [1911] 1981, pp. 93-103.

, A Room of One's Own. London: Grafton Books, [1929] 1987.

"Women and Fiction" ([1929]). En: Deborah Cameron (ed.) The Feminist Critique of Language. A Reader. London: Routledge, 1990, pp. 33-40.

, Collected Essays. London, Chatto \& Windus, 1967. 
Hikma 7 (2008), 9-23 